\title{
Computation of magnetic field in an actuator
}

\author{
A. G. Olabi and A. Grunwald \\ Dublin City University, School of Mechanical and Manufacturing Engineering, Glasnevin, \\ Dublin 9, Ireland, Email: abdul.olabi@dcu.ie, artur.grunwald@dcu.ie
}

\begin{abstract}
:
Design and optimization of an actuators based on magnetostrictive technology requires computation of the magnetic field. The "MS"-technology offers an attractive controllability with high power density. The magnetostriction is a reversible feature which can be used in various actuator layouts. The actuator performance depends on driving magnetic field and the particular magnetic properties of used materials. Good understanding of specific design constrains is required to define and to optimized a magnetostrictive actuator. The non-linear computation of the magnetic field using FEM software is vital for the finale experimental design of a low-frequency actuator. This paper presents results of magnetic field simulation with FEMM software package and experimental measurements of the magnetic flux density. Good correlation between the simulation results and experimental measurements has been achieved.
\end{abstract}

Keywords: FEM, FEMM, Magnetostriction, Actuator, Terfenol-D 


\section{Introduction of the magnetostrictive actuator}

Magnetostriction ("MS") is the change in shape of materials under the influence of an external magnetic field. The magnetostrictive effect was first described in the $19^{\text {th }}$ century (1842) by an English physicist James Joule. Several applications based on magnetostrictive technology have been introduced in various industries. A summary of literature survey can be found in article [1]. The article [2] captures the optimization of the strain performance using mechanical pre-stress. The maximum useful magnetoelastic strain is one of the key parameters defining the resulting mechanical output in the case of a magnetostrictive actuator. In case a shaft is made of magnetostrictive material, i.e. Terfenol-D, magnetic field along the shaft axle will cause axial elongation. The applied magnetic field leads to relative strain in the magnetostrictive material. A higher magnetic field causes higher strain and leads to larger elongation. Without the magnetic field the shape of the magnetostrictive material reverse to the original. Fig. 1 depicts a cross section of the actuation hardware based on magnetostrictive technology.
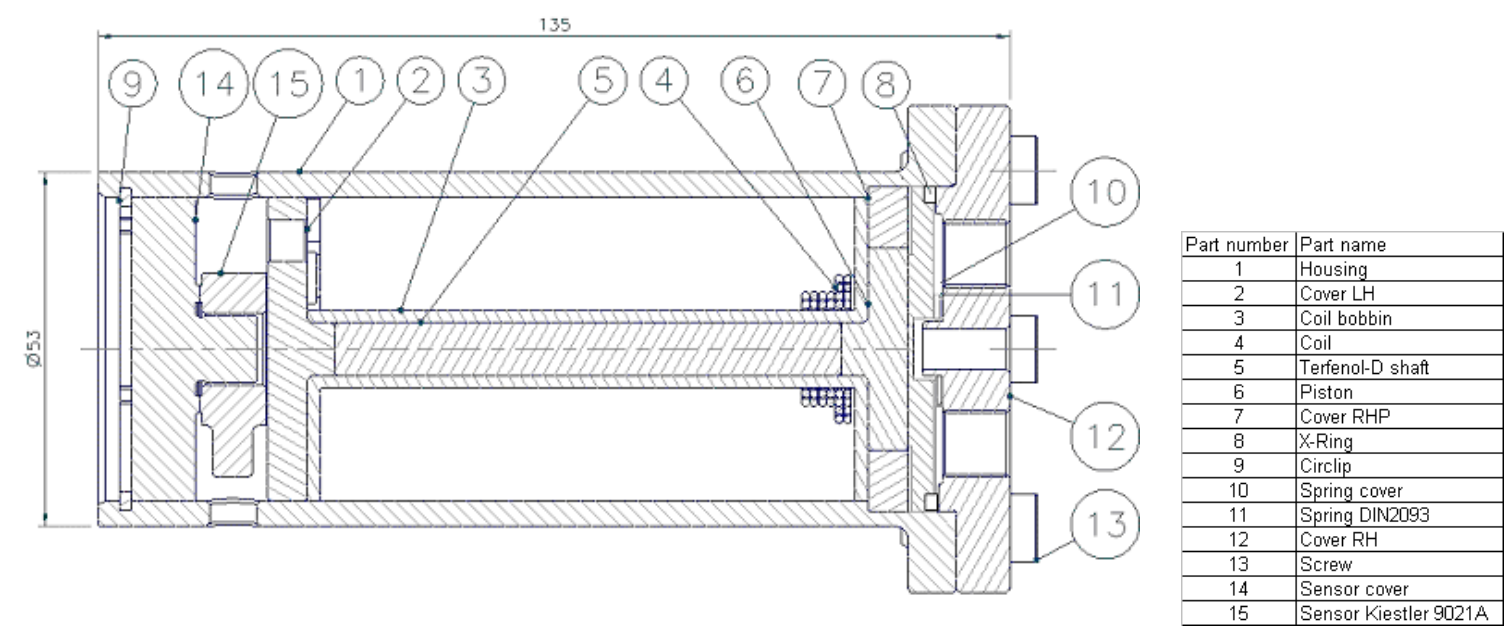

Fig. 1: Cross-section of the actuator used in the study

\section{The Magnetic circuitry for the magnetostrictive actuator}

A coil in an appropriate ferromagnetic housing is defined as the source of the magnetic field. The chosen diameter of the Terfenol-D shaft is $8 \mathrm{~mm}$ and the length is about $68 \mathrm{~mm}$ [2]. The magnetic field is generated by electric current $\mathrm{I}[\mathrm{A}]$ through the actuator coil. The coil is wound around the Terfenol-D shaft and the magnetic field is therefore parallel to the axis of the rod. Fig. 2 depicts the generally the coil layout. 


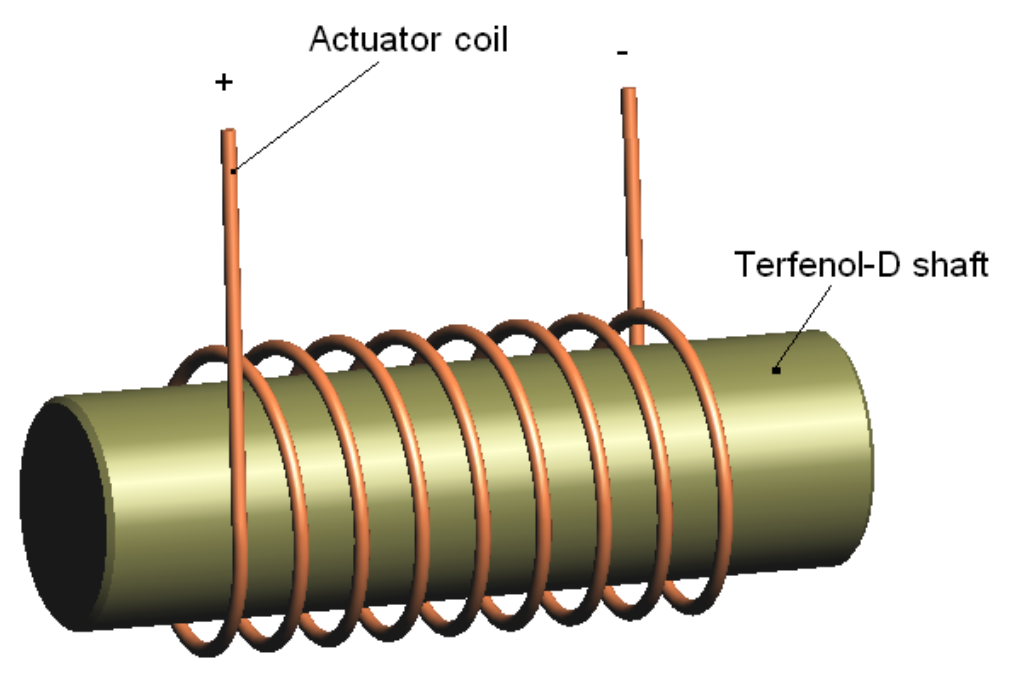

Fig. 2: Layout of the actuator coil

Since not only the coil is involved in the magnetic circuit other ferromagnetic components like housing, Terfenol-D shaft and inserts have to be considered. Fig. 3 presents the main magnetic path through the magnetostrictive actuator.

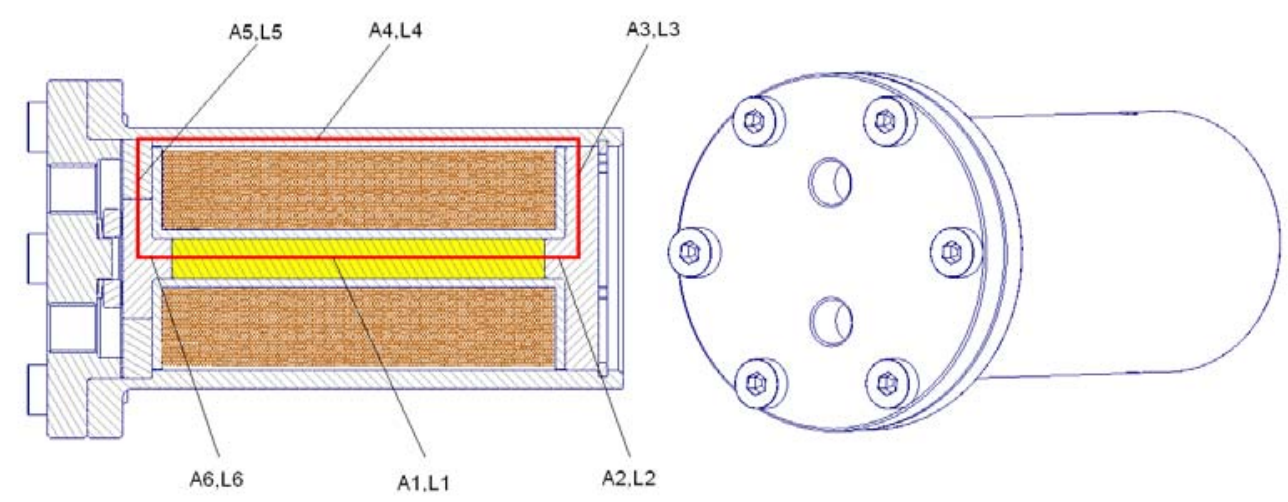

Fig. 3: Magnetic path through the actuator

In Fig. 3 there are six designated sections of component through which the magnetic flux passes. It is important to include all six terms in order to estimate the required total magnetomotive force, and so the return path of the magnetic flux through steel components must also be considered. For each section there is a length of the magnetic path, l, and a value for the magnetic field strength, $H$. The length is fixed by the geometry of the system, but the value of $\mathrm{H}$ must be determined by making use of the magnetic properties of the material. Fig. 4 presents the coil specification which has been used for the magnetic field simulation and for the experimental evaluation. 


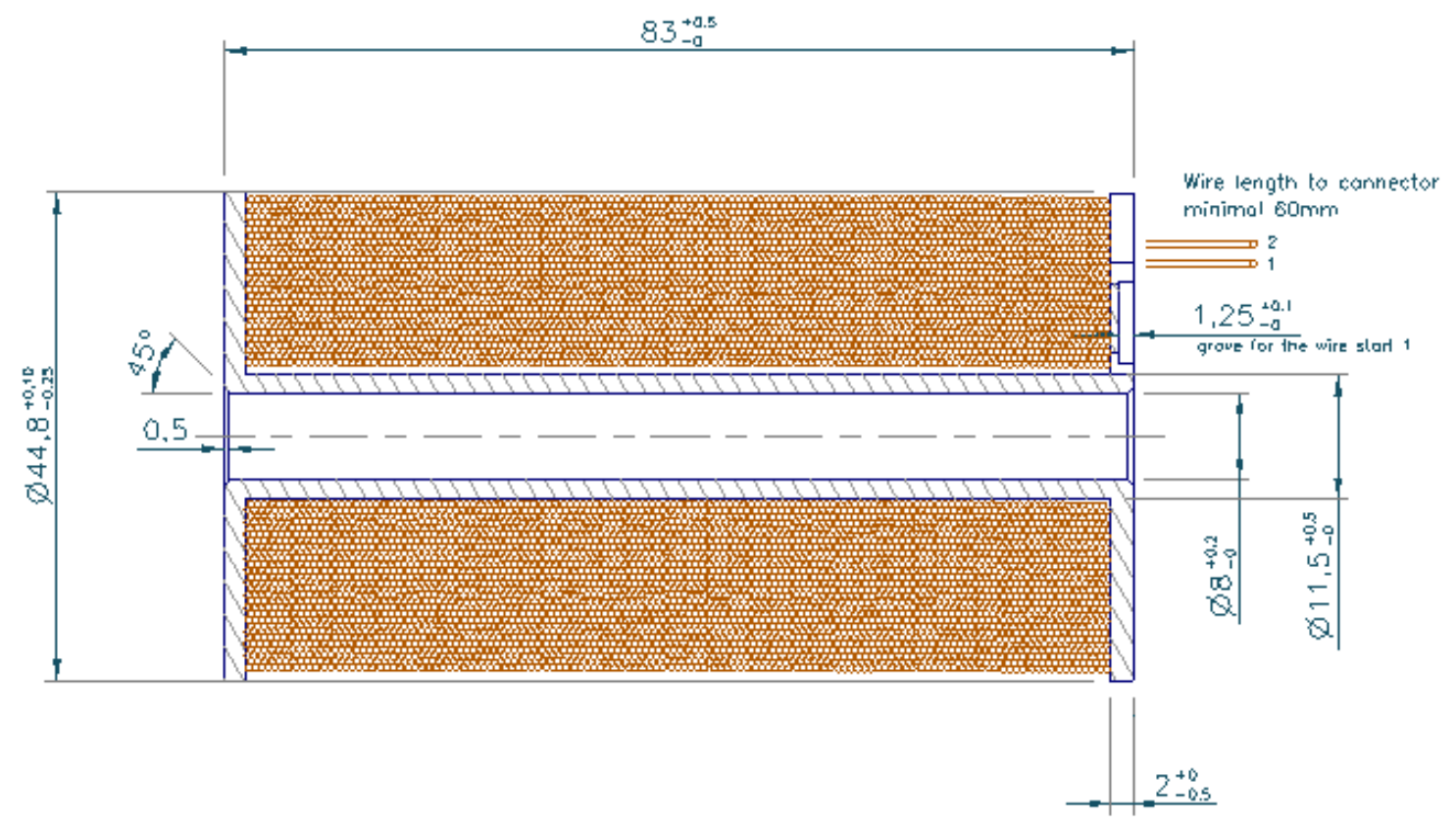

Actuotor coil for rig tests

Temperature range: $-20 \mathrm{C}$ up to $+120 \mathrm{C}$

Supplied voltage: $12 \mathrm{~V}+/-3 \mathrm{~V}$.

Frequency: $\mathrm{OHz}$ at $\mathrm{DC}$ and $100 \mathrm{OHz}$ at $\mathrm{AC}$

Resistonce ot Rt: $>1.52$ ohm ot DC

Inductance: $23 \mathrm{mH}$

Nominal current: $<9.8$ A

Number of turns: $>1000$

Wire moterial: Copper

Wire bore diameter: $1.15 \mathrm{~mm}$ (AWG17)

Fig. 4: Electric coil for the actuator

In order to verify the simulated model of actuator, the experimental rig assembly has been prepared for measurements of flux density with Tesla Meter. The magnetic properties of Terfenol-D at various pre-stress levels have been provided by the Terfenol-D supplier [3]. Fig. 5 depicts the B-H characteristic of the Terfenol-D shaft at pre-stress about 7.2MPa.

The pre-stress in the assembly has been achieved by using appropriate preload-spring (part number 11 in Fig. 2). A force sensor based on piezo technology (part number 15 in Fig. 2) has been used to measure the pre-load in the experimental rig assembly. 
B-H Diagram Terfenol-D (7.2MPa)
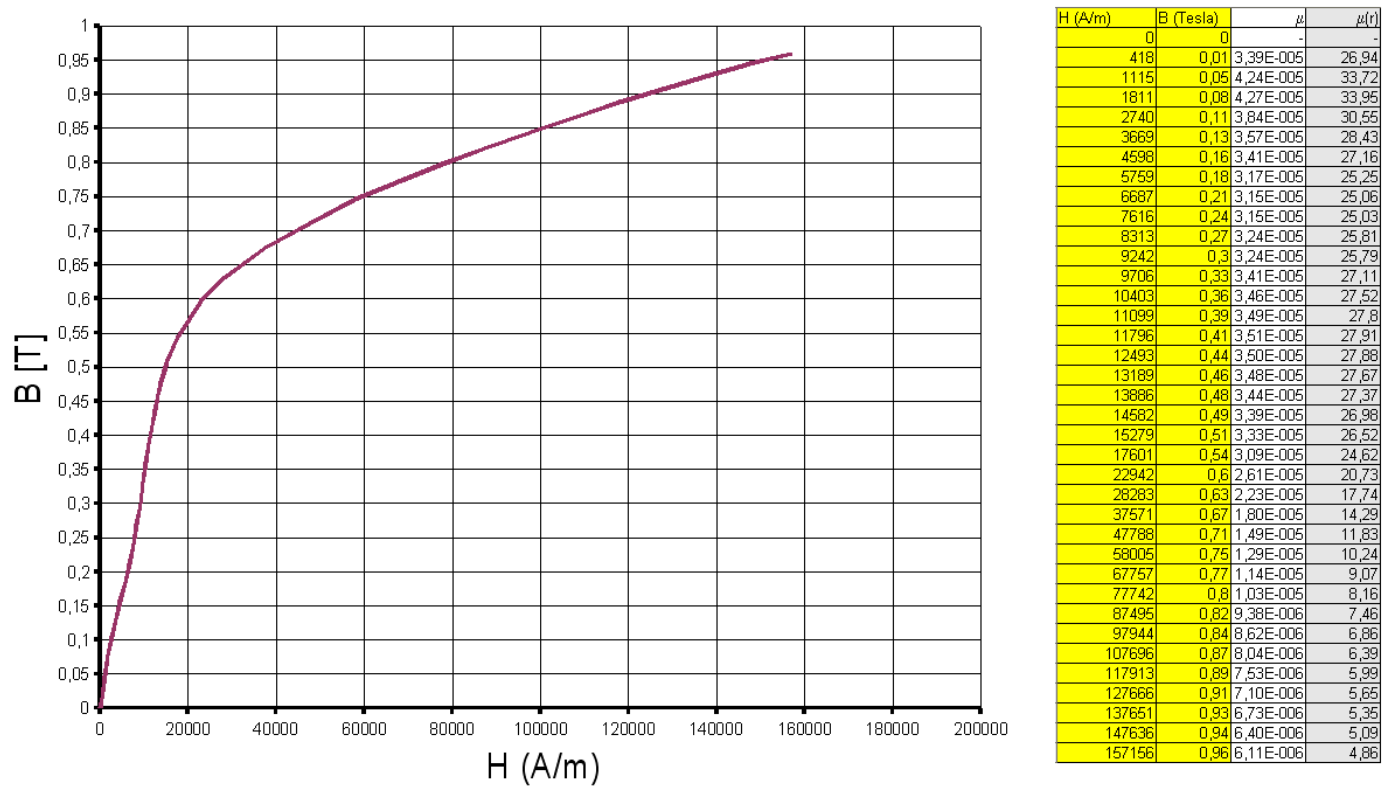

Fig. 5: B-H diagram of Terfenol-D with measurement data [3]

For the housing of the actuator the low carbon steel, Ck15, has been used. The magnetic properties of Ck15 are shown in Fig. 6.

B-H Diagram Ck15
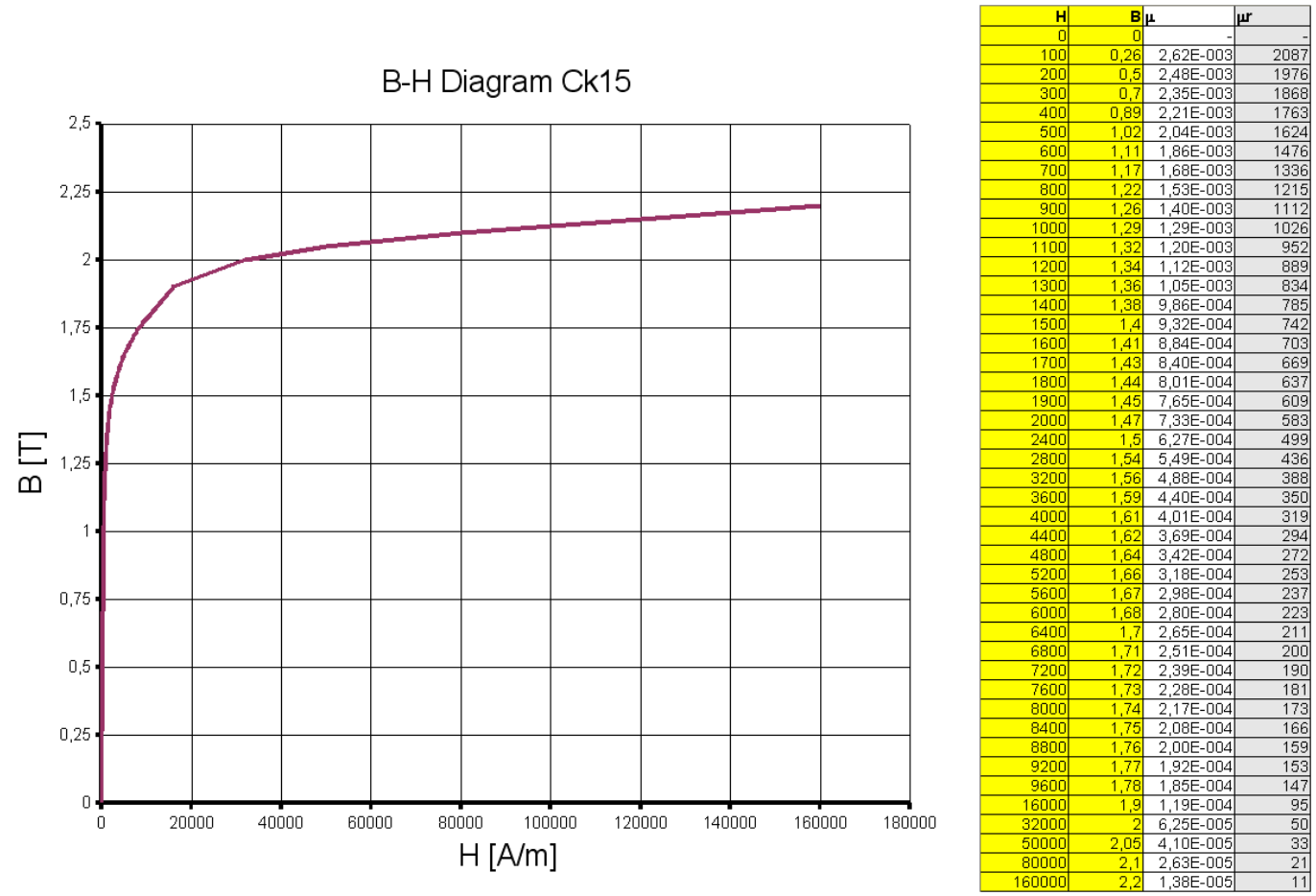

Fig. 6: B-H diagram of Ck15 with measurement data 


\section{Magnetic Field Simulation}

In order to optimize the design of the actuator, a simulation of magnetic field, including the real magnetic properties of used material, is required. The nonlinear simulation method was used to determine and optimize the actuator and control performance. Due to nonlinear B-H function the system has to be solved by an iterative way. Finite element modelling methods of the magneto-mechanical phenomena have been proposed in several publications. Terfenol$\mathrm{D}$ is a smart material in that the magnetic properties are coupled with mechanical state and vice versa. For the optimization of the proposed structure the free available software package FEMM have been used. The simple user interface and efficiency has been found in using the free available FEMM software packaging developed by David Meeker, Senior Engineer at Forster-Miller Inc. [4]. Results from the magnetic field simulation from FEMM have been evaluated and positively verified with measurements. For low-frequency $(<500 \mathrm{~Hz})$ evaluations only a part of the complete Maxwell's equations is considered. Fig. 7 shows the reference plot of the cross-section from the actuator.

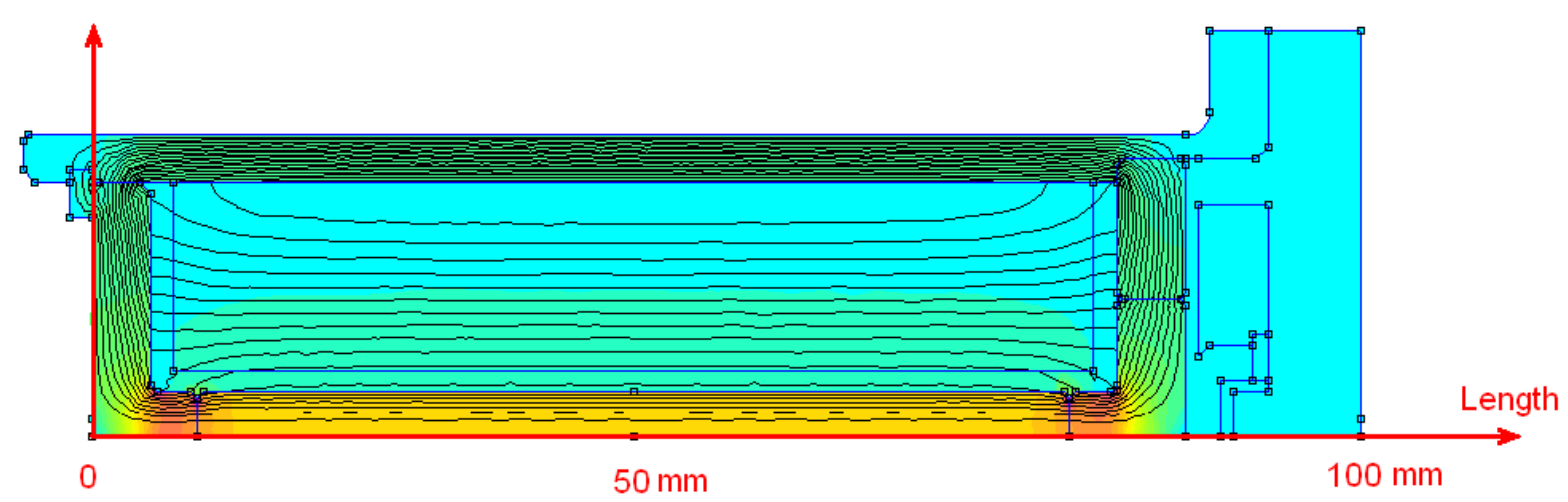

Fig. 7: Reference figure of the actuator plots of $B(T)$ and $H(A / m)$

Due to proposed measuring method with the Tesla-Meter, minor modifications of the actuator structure were required. Following simulation model has been used to verify the simulated flux density with the measured flux density. Fig. 8 presents the actuator model without (left) and with (right) Tesla-Meter probe. 

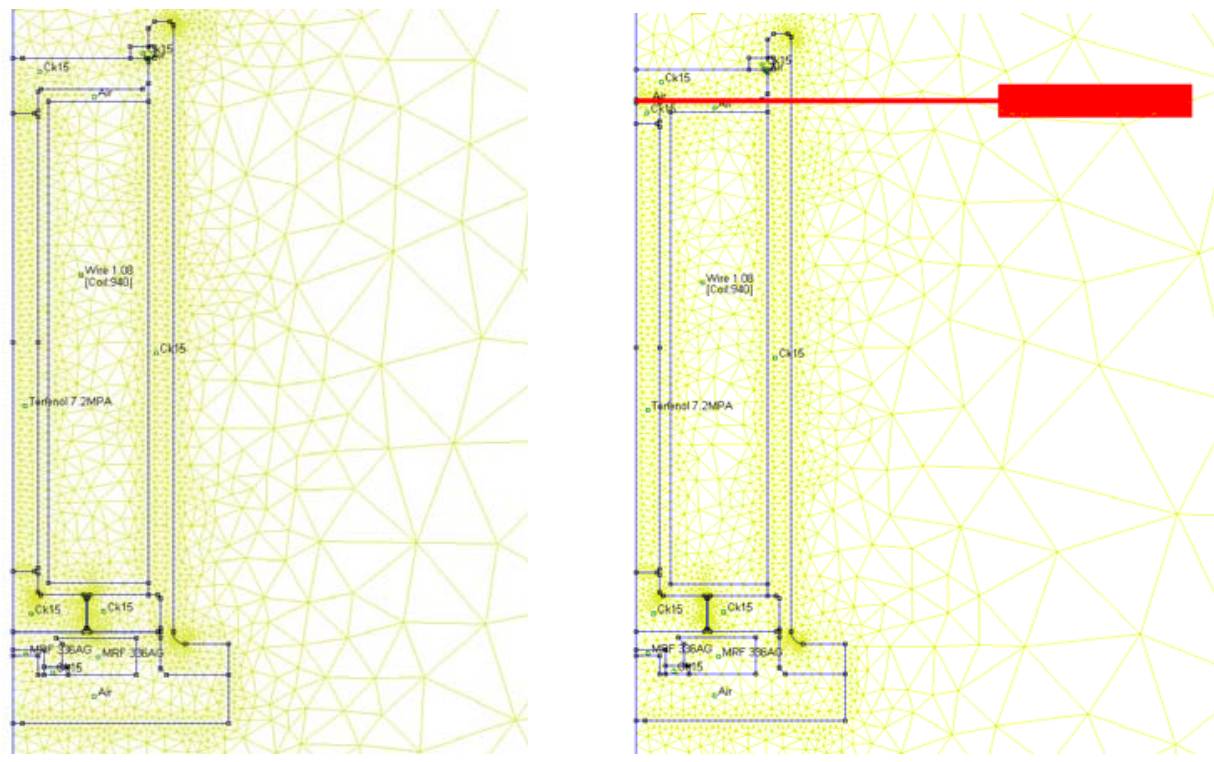

Fig. 8: Actuator model without (left) and with (right) Tesla-Meter probe

Fig. 9 presents the simulation results of flux density B (T) obtained with FEMM at nominal electrical current of about 9A. On the left side: the original actuator assembly; on the right side: the modified assembly for Tesla-Meter probe. Top-to-bottom reference centre line has been used as reference.
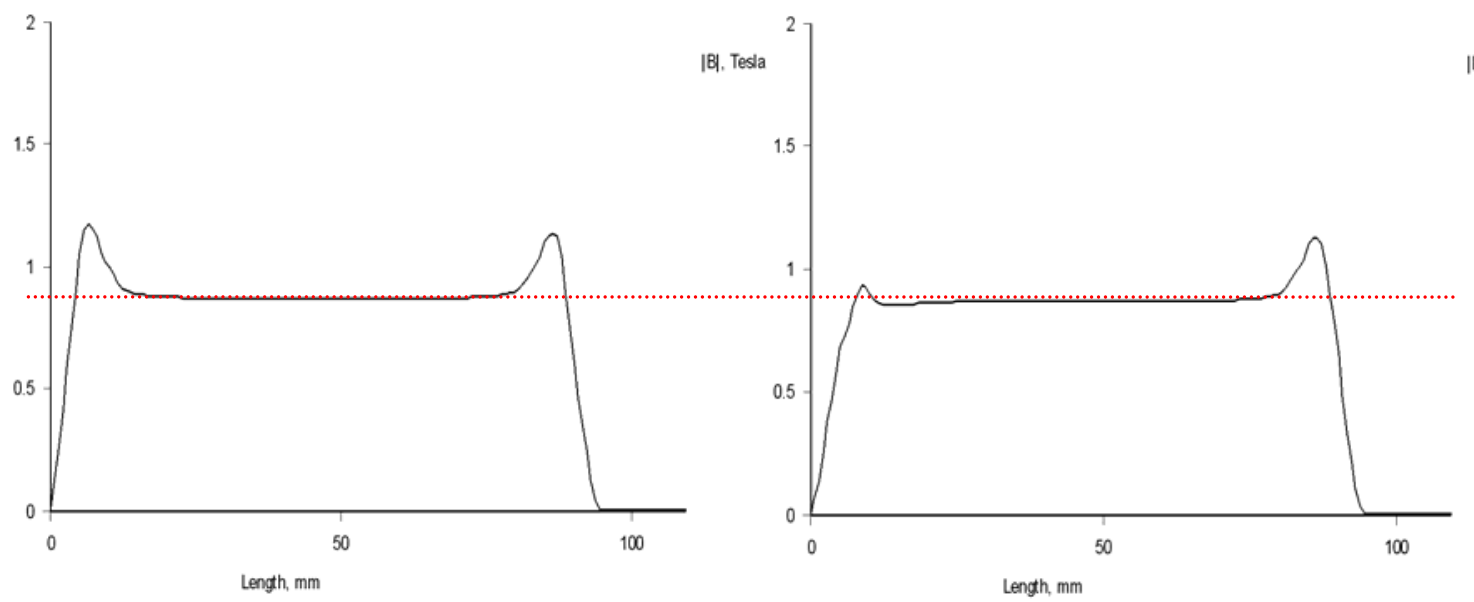

Fig. 9: Flux density without (left) and with (right) Tesla-Meter probe

The difference between the two above showed results has been found as acceptable for verification of the magnetic field simulation results. Figures 10 and 11 show some simulation results of flux density $\mathrm{B}(\mathrm{T})$ and field intensity $\mathrm{H}(\mathrm{kA} / \mathrm{m})$ at various current levels. 


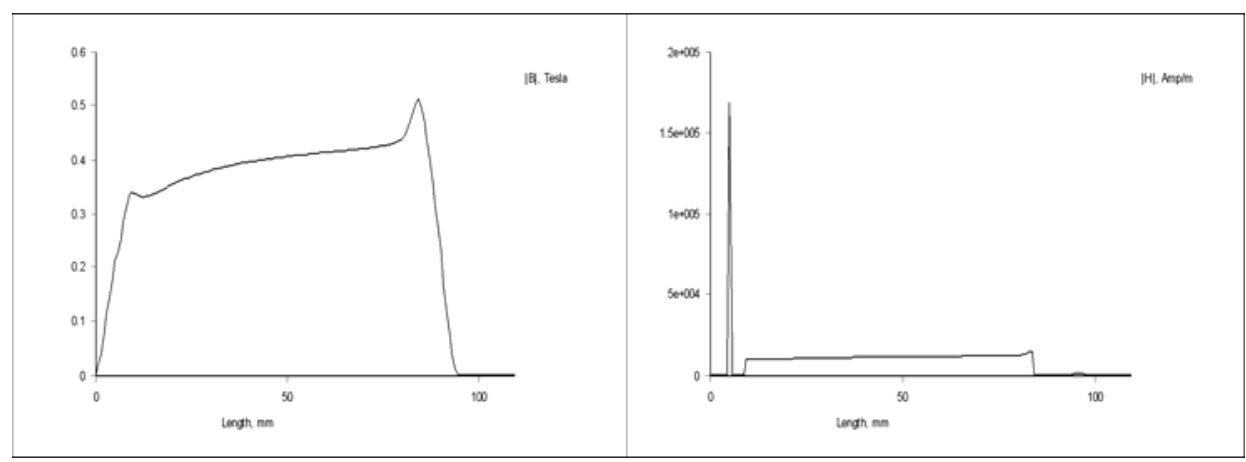

Fig. 10: FEMM flux density B (left) and field intensity $\mathrm{H}$ (right) at $1 \mathrm{~A}$

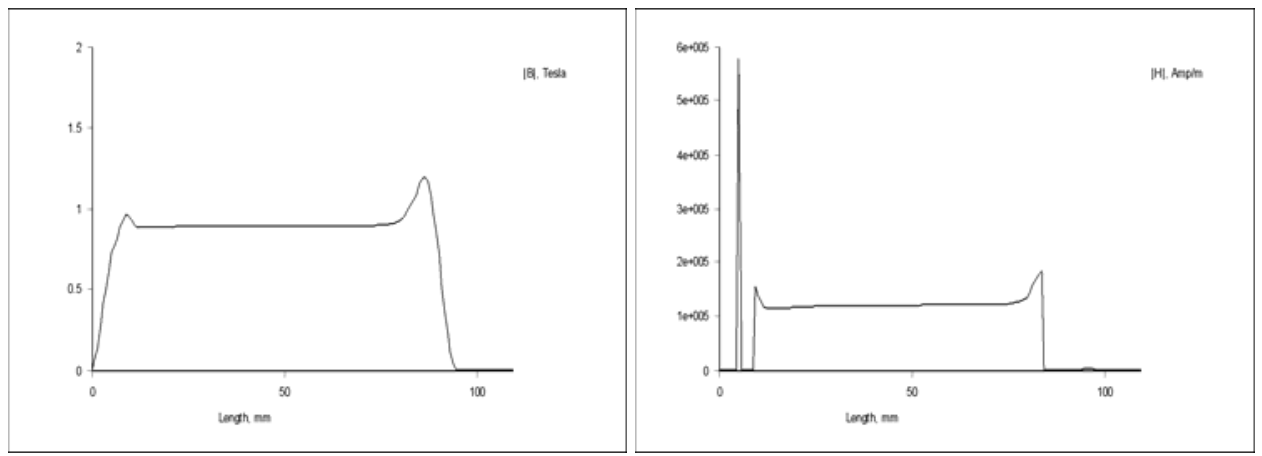

Fig. 11: FEMM flux density B (left) and field intensity $\mathrm{H}$ (right) at $10 \mathrm{~A}$

\section{Experimental evaluation}

A power supply unit (up to 10A, DC), a Multi-Meter for current measurements and the TeslaMeter Model 5080 have been used for experimental evaluation. The actuator assembly has been completed and used to verify the simulation results with measured magnetic flux density. Fig. 12 depicts the test bench layout for magnetic flux density measurements.

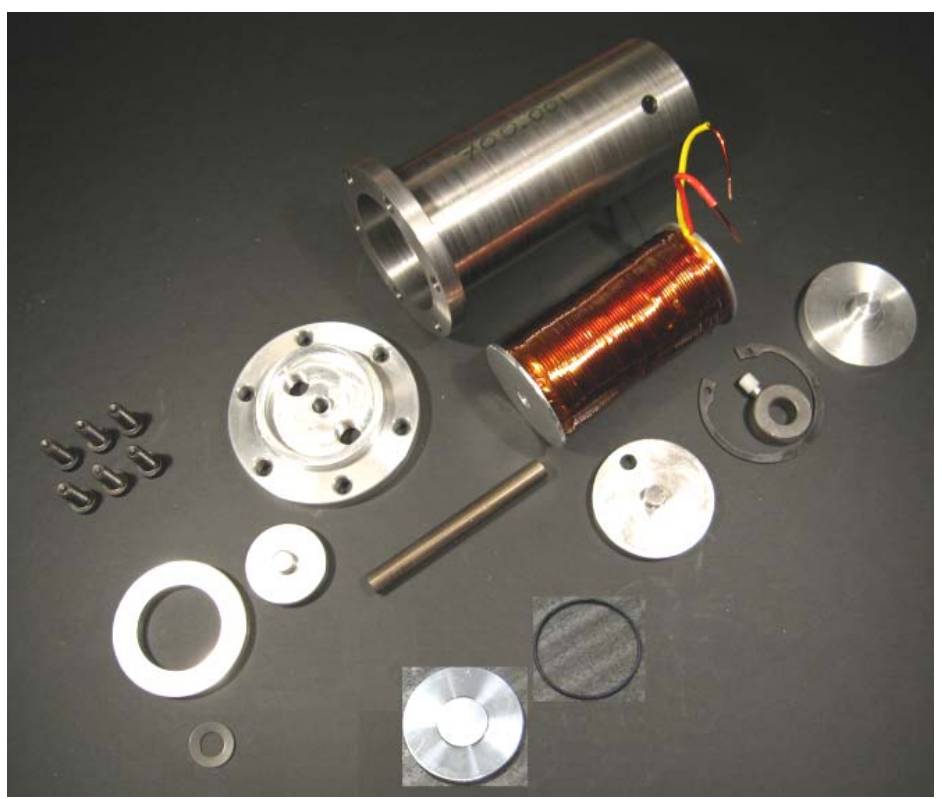

Fig. 12: Picture from “MS”-actuator assembly 
The flat Tesla-probe has been adapted in to the actuator assembly according Fig. 13.

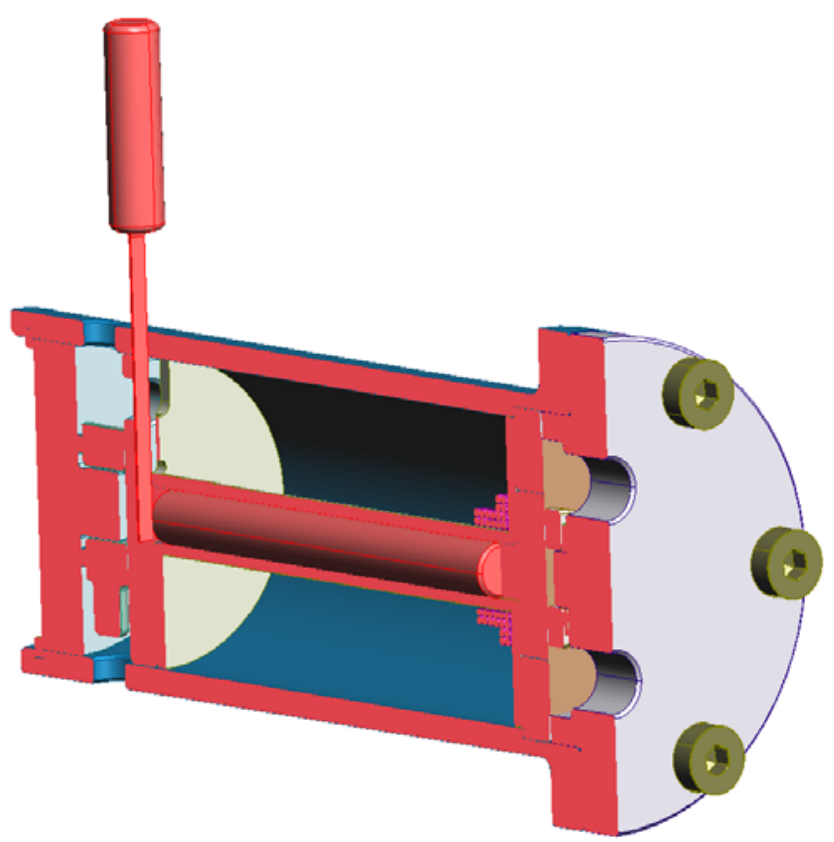

Fig. 13: Actuator with Tesla-Meter probe (Model 5080)

The Fig. 14 presents the Tesla-Meter with the transverse probe. Hall effect element at the top of the flat probe has been used to measure the magnetic flux density B in tesla.
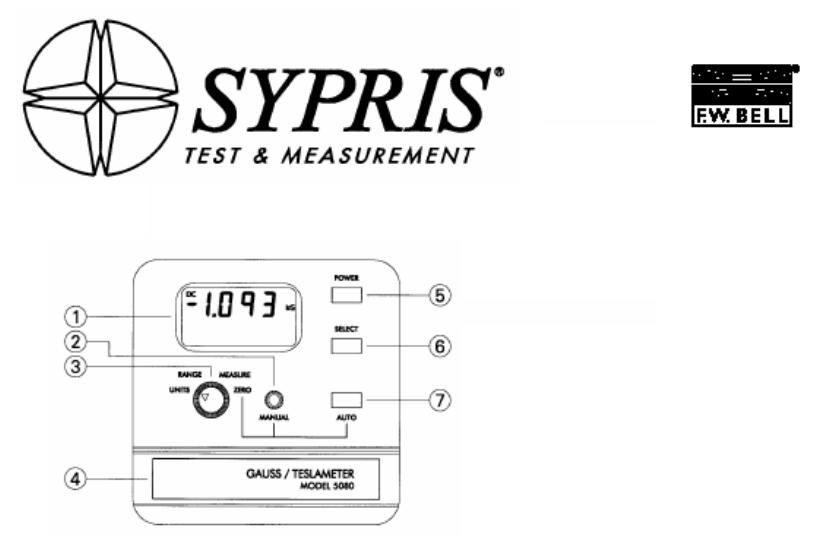

ACCURACY (analog output, including probe)

dc mode: $\quad \pm 1 \%$ of reading, $\pm 5 \mathrm{mV}$.

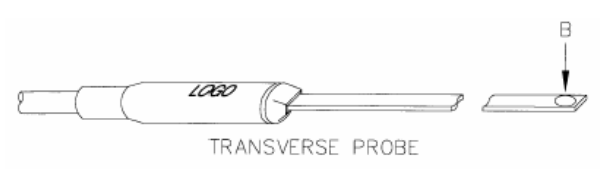

\section{Model 5080}

GAUSS / TESLA METER

Fig. 14: Tesla-Meter Model 5080 [5] 
Table 1 presents the results from FEMM magnetic field simulation and the measurement results of flux density at the top of the Terfenol-D shaft

Table 1: Simulation and measurement result for comparison

\begin{tabular}{|c|c|c|c|c|}
\hline $\begin{array}{c}\text { Electric current I } \\
(\text { Amp) }\end{array}$ & $\begin{array}{c}\text { FEMM flux density } \\
\text { in the gap B (Tesla) }\end{array}$ & $\begin{array}{c}\text { Measured flux density } \\
\text { in the gap B (Tesla) }\end{array}$ & $\begin{array}{c}\text { FEMM flux density in } \\
\text { Terfenol-D B (Tesla) }\end{array}$ & $\begin{array}{c}\text { Expected field intensity in } \\
\text { Terfenol-D H (A/m) at 7.2MPa }\end{array}$ \\
\hline 0.0 & 0.000 & 0.000 & 0.000 & 0 \\
\hline 1.0 & 0.200 & 0.220 & 0.400 & 11800 \\
\hline 2.0 & 0.330 & 0.300 & 0.600 & 23000 \\
\hline 3.0 & 0.400 & 0.342 & 0.660 & 37000 \\
\hline 4.0 & 0.450 & 0.382 & 0.710 & 47800 \\
\hline 5.0 & 0.500 & 0.417 & 0.750 & 58000 \\
\hline 6.0 & 0.540 & 0.450 & 0.775 & 67800 \\
\hline 7.0 & 0.580 & 0.480 & 0.810 & 87000 \\
\hline 8.0 & 0.620 & 0.520 & 0.830 & 93000 \\
\hline 9.0 & 0.640 & 0.560 & 0.870 & 108000 \\
\hline 10.0 & 0.660 & 0.580 & 0.890 & 118000 \\
\hline
\end{tabular}

Fig. 15 depicts the measured flux density at the top of the Terfenol-D shaft with simulated results, obtained with FEMM.

Magnetic field flux density (B) and field intensity $(\mathrm{H})$ versus current I

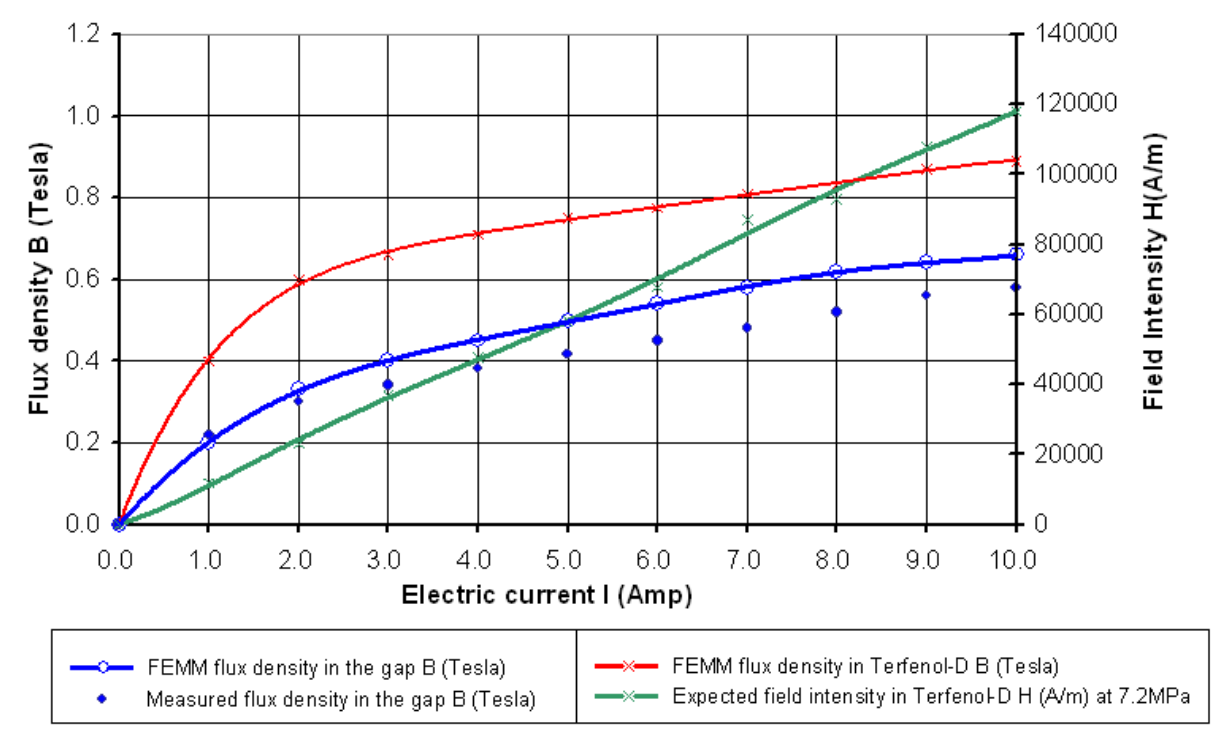

Fig. 15: FEMM flux density B (T) versus electrical current

\section{Conclusions}

Summarizing the results from simulation and the flux density measurement with the TeslaMeter Model 5080 can be stated, that the results are within the specified range and in good consistence. The FEMM software for non-linear magnetic field simulation can be used to finalize and to optimize the product design. The difference in the simulated results and the measured flux density is predictable and was caused by accepted variations of the housing material and measurement tolerances. The presented simulation results have been used for design freeze and for further experimental evaluations of the magnetostrictive actuator. 


\section{Reference list}

[1] A. G. Olabi, A. Grunwald, Design and application of magnetostrictive materials, Materials And Design, 29, 2, pp469-483

[2] A. G. Olabi, A. Grunwald, Design of magnetostrictive (“MS”) actuator, Sensors and Actuators A: Physical, Volume 144, Issue 1, 28 May 2008, Pages 161-175.

[3] ETREMA Products Inc., Terfenol-D Magnetostrictive Actuator Information, Specifications, Public domain information, www.terfenoltruth.com, www.etrema.com [4] D. Meeker, FEMM-Software Manual, Finite Elements Method Magnetics User's Manual and Tutorial, 2004, http://femm.foster-miller.net/wiki/FAQ

[5] Tesla-Meter Instruction Manual, F.W. Bell, Tesla-Meter Model 5080 